\title{
Inverse Association between Circulating Levels of Soluble Receptor for Advanced Glycation End-Products and Coronary Plaque Burden
}

\author{
Giuseppina Basta ${ }^{1}$, Serena Del Turco ${ }^{1}$, Teresa Navarra ${ }^{1}$, Alessandro Mazzarisi ${ }^{1}$, Franca Cocci ${ }^{2}$, \\ Michele Coceani ${ }^{2}$, Massimiliano Bianchi ${ }^{2}$, Mathis Schlueter ${ }^{2}$ and Paolo Marraccini ${ }^{1}$
}

${ }^{1}$ CNR Institute of Clinical Physiology, Pisa, Italy

${ }^{2}$ Fondazione G. Monasterio CNR-Regione Toscana, Pisa, Italy

\begin{abstract}
Aim: Low levels of soluble receptor for advanced glycation end-products (sRAGE) have been reported to be associated with coronary artery disease (CAD) and peripheral atherosclerosis. This study explored the relationship between circulating levels of sRAGE and the characteristics of coronary vessels detected by 64-slice computed tomography angiography (CTA).

Methods: In this cross-sectional study we included 127 consecutive patients with CAD but without acute coronary syndrome. Quantitative volumetric analysis of the lumen and plaque burden of the vessel wall (soft and calcific components) was performed for the three major coronary vessels. Each component was expressed as a percentage of vessel volume and utilized in per-patient analysis. The patients were classified into two groups according to the presence of calcium volume: non-calcified plaque $(\mathrm{NCP})$ group (calcium volume $\%=0$ ) and calcified plaque $(\mathrm{CP})$ group (calcium volume $\%>0$ ). Results: In the NCP group, but not in the CP group, simple regression analysis revealed a negative association of total plaque burden \% with sRAGE $(\beta=-0.378, p=0.0019)$ and HDL cholesterol $(\beta=-0.368, p=0.003)$ and a positive association with creatinine $(\beta=0.258, p=0.041)$ and male gen$\operatorname{der}(\beta=0.317, p=0.01)$. After adjusting for confounding factors, the total plaque burden $\%$ remained significantly associated only with $\operatorname{sRAGE}(\beta=-0.358, p=0.011)$.

Conclusions: Circulating sRAGE levels are associated in an inverse manner with non-calcified plaque burden, suggesting that it may be related with early atherosclerosis and plaque progression.
\end{abstract}

J Atheroscler Thromb, 2012; 19:941-948.

Key words; 64-slice computed tomography angiography, Soluble receptor for advanced glycation endproducts, Coronary artery disease

\section{Introduction}

The receptor for advanced glycation end-products (RAGE) is a multi-ligand receptor that contributes to the pathogenesis of tissue injury in metabolic disorders and aging through innate and adaptive inflammatory mechanisms ${ }^{1-3)}$.

In recent years, the circulating isoform of RAGE, called soluble RAGE (sRAGE), which is able to competitively inhibit ligand binding to membrane-bound

Address for correspondence: Giuseppina Basta, CNR, Institute of Clinical Physiology, San Cataldo Research Area, Via Moruzzi,

1, 56124 Pisa, Italy

E-mail: lapina@ifc.cnr.it

Received: June 14, 2011

Accepted for publication: May 8, 2012
RAGE, was reported as an emerging biomarker in cardiovascular, metabolic and inflammatory diseases ${ }^{4,5)}$.

sRAGE, consisting of the extracellular ligandbinding domain only, lacking both cytoplasmic and transmembrane domains, is generated by alternative splicing or proteolytic cleavage of full-length RAGE ${ }^{2)}$. It circulates in human plasma and is still able to bind ligands and thus antagonize RAGE signalling ${ }^{4,5)}$. This hypothesis is supported by animal testing, where administration of sRAGE to mouse models retarded the progression of atherosclerosis, and by clinical studies, where low sRAGE levels were reported to be linked to the presence of angiographically proven coronary artery disease $(\mathrm{CAD})^{6}$, , cardiovascular events ${ }^{7)}$ and carotid intima media thickness ${ }^{8,9)}$, suggesting a vascular-protective role for this molecule. 
Multi-slice computed tomography angiography (CTA) is a non-invasive tool that produces high-resolution imaging of the coronary artery wall and allows more reliable detection of both obstructive and nonobstructive subclinical CAD at an earlier stage than by invasive angiography ${ }^{10-12)}$. CTA permits the detection of both calcified plaque and non-calcified coronary atherosclerotic plaque with good agreement with intravascular ultrasound ${ }^{13)}$.

\section{Aim}

To assess the relationship between sRAGE plasma levels and the characteristics of coronary vessels detected by CTA in a cohort of subjects who presented with chest pain syndrome and in whom acute coronary syndrome (ACS) had been excluded.

\section{Methods}

\section{Study Population}

From November 2007 to July 2009 we recruited 200 consecutive patients (age 63.3 \pm 12 years; $63.5 \%$ men) with chest pain syndrome, proven or suspected CAD who had been screened with 64-slice CTA according to guidelines ${ }^{14)}$.

The exclusion criteria were as follows: patients who were either suspected of having ACS or had severe symptoms before CTA $(n=10)$, patients with chronic total occlusion $(n=13)$, elevated pre-procedural cardiac biomarkers $(n=14)$, previous stenting $(n=23)$, and inestimable-quality CTA imaging $(n=$ 13). Thus, the final study population was 127 patients.

The study protocol was approved by the institutional ethics committee and all patients provided informed consent to the study.

\section{Cardiovascular Risk Factors}

We obtained information on and clinical measurements of risk factors and medication during the patient's visit. Hypertension was defined as systolic blood pressure over 140 and/or diastolic blood pressure over $90 \mathrm{mmHg}$ and/or treatment with antihypertensive medication. Antihypertensive therapy included the following classes of drugs or any combination of them: ACE inhibitors, calcium-antagonists, betablockers, and diuretics. Platelet aggregation inhibitors, such as aspirin $(<500 \mathrm{mg} /$ day $)$, were recorded.

Hypercholesterolemia was defined as fasting cholesterol over $200 \mathrm{mg} / \mathrm{dL}$ or use of cholesterol-lowering drugs (statins, fibrates, bile acid sequestrants and nicotinic acid derivatives). Diabetes was defined as fasting serum glucose levels over $126 \mathrm{mg} / \mathrm{dL}$ or use of antidia- betic medication (insulin or any oral antidiabetic medication). Subjects were classified as smokers if they had smoked at least one cigarette per day in the year before the study. Information was collected on previous cardiovascular and cerebrovascular events. A family history of CAD was defined as having a first-degree female ( $<65$ years) or male $(<55$ years) relative with a documented history of myocardial infarction (MI), sudden cardiac death and surgical or percutaneous coronary revascularization.

The Framingham risk score was calculated for each subject using the risk score of Wilson et al. ${ }^{15)}$.

\section{Laboratory Assays}

Blood samples were drawn before the CTA procedure and collected in tubes without additives, containing heparin or citrate (for routine biochemistry). Total cholesterol, high density lipoprotein (HDL) cholesterol, triglycerides, and glucose were determined using routine laboratory procedures. Plasma and serum samples were centrifuged at $4{ }^{\circ} \mathrm{C}$, immediately divided into aliquots and stored at $-80^{\circ} \mathrm{C}$ until analysis. All laboratory determinations were performed in a blinded fashion.

\section{sRAGE Assay}

Blood samples were collected in tubes containing $\mathrm{Na} 2 \mathrm{EDTA}$, and sRAGE levels were determined using a double-sandwich ELISA kit (DuoSet ELISA development kit; R\&D Systems, Minneapolis, MN) as described previously ${ }^{9)}$. The intra-assay and inter-assay coefficients of variation were $5.9 \%$ and $8.2 \%$, respectively. The lower limit of sRAGE detection was 21.5 $\mathrm{pg} / \mathrm{mL}$.

\section{4-Slice CTA Scan}

The patients underwent 64-slice CTA (LightSpeed VCT 64, GE Healthcare, Milwaukee, WI) with the following scan parameters: retrospective ECG gating; 912 channel detectors along the gantry and 64 channel detectors along the z-axis; tube voltage 120 $\mathrm{kV}$; tube current 350-750 mA (depending on patient size); scan FOV $50 \mathrm{~cm}$; gantry rotation $0.35 \mathrm{~s} /$ rotation; matrix $512^{\circ}-512$; slice thickness $0.625 \mathrm{~mm}$; range of helical pitch $0.18-0.24$. When appropriate the following premedications were administered: metoprolol, up to $5 \mathrm{mg}$ intravenously, to lower the heart rate below 65 beats per minute; isosorbide dinitrate, up to $1 \mathrm{mg}$ intravenously, to guarantee maximal epicardial vasodilatation. Non-ionic iodinated contrast medium (Iomeprol 400; Bracco Imaging SpA, Milan, Italy) was injected via a peripheral vein according to a triphasic protocol using a programmable injector 
(Nemoto Dual Shot Injector; Nemoto Kyorindo Co. Ltd., Tokyo, Japan) with a two-way syringe system: in the first phase, rapid injection of contrast medium (from 5 to $8 \mathrm{~mL} / \mathrm{sec}$ ) was performed; in the second phase, $10 \mathrm{~mL}$ contrast at $1 \mathrm{~mL} / \mathrm{sec}$ was injected simultaneously with $25 \mathrm{~mL}$ saline at $2.5 \mathrm{~mL} / \mathrm{sec}$; in the third phase, $35 \mathrm{~mL}$ saline flush was administered at 4 $\mathrm{mL} / \mathrm{sec}$ (maximal total volume of contrast medium $110 \mathrm{~mL}$ ). To time the scan, a region of interest (ROI) was placed in the right ventricular cavity to detect peak enhancement. Scans were performed during breath hold; patients were monitored continuously through single-lead electrocardiography. The scan parameters were programmed in order to limit radiation exposure to $15 \mathrm{mSv}$ on average. After the procedure, patients had an intravenous infusion of saline $(500 \mathrm{~mL})$ to improve hydration and prevent contrastinduced nephropathy. Moreover, all patients were instructed to repeat the measurement of serum creatinine between 2 and 7 days after the examination.

\section{Image Reconstruction and Analysis}

Trans-axial CT images were reconstructed using a slice thickness of $0.625-\mathrm{mm}$ and $0.4-\mathrm{mm}$ increments. The data were then transferred to a dedicated workstation (Advantage Workstation 4.3; GE Healthcare) for post-processing. Lumen size (diameter and area) of the major coronary arteries was measured using "Multiplanar Reformatting Images" reconstruction with an automatic interactive program. Lumen reduction $\geq 50 \%$ was classified as significant stenosis. Volumetric analysis of the coronary vessel wall was evaluated using Color Code Plaque analysis software (GE Healthcare) ${ }^{16)}$. This densitometric method, based on the Hounsfield scale, allows the computation of a cylindrical volume around the vessel lumen and the relative contribution of calcium, fibro-fatty and fibromuscular components of the vessel wall. The algorithm of vessel wall analysis was tuned in each patient according to densitometric parameters measured by a ROI on the aortic root, left ventricular myocardium and epicardial fat. Using this system, CT density $<60$ HU was attributed to the fibro-fatty component, between 60 and $200 \mathrm{HU}$ to the fibro-muscular component, while calcified plaques were differentiated from the lumen when they had a density $>800 \mathrm{HU}$. The program output gave a quantitative volumetric measurement $\left(\mathrm{mm}^{3}\right)$ of each component. In this paper we considered the total plaque burden as the sum of the soft component (defined as fibro-fatty and fibrotic component) and calcified component in the three major coronary vessels. Each component was also expressed as a percentage of vessel volume and utilized in per-patient analyses.

\section{Statistical Analysis}

Data were analyzed with the use of statistical software SPSS 13.0 (SPSS Inc., Chicago, IL).

The Kolmogorov-Smirnov test of normality was used to verify whether the distribution of variables followed a Gaussian pattern. Data with a normal distribution are given as the mean \pm SD. Variables with a skewed distribution are expressed as the median and interquartile range. Group differences were analyzed by Student's $t$-test, and the $\chi^{2}$ test for normally distributed and non-continuous variables, respectively. Variables with a non-normal distribution were logarithmically transformed before each analysis.

Simple regression analysis and multivariate linear regression analysis were used to test the relationship among clinical, laboratory, and CTA parameters. Traditional risk factors and factors with $p<0.10$ on univariate analysis were entered into multiple regression analysis. A two-tailed $p$-value $<0.05$ was considered significant.

\section{Results}

A total of 381 major coronary vessels were evaluated in 127 patients. Coronary calcification (calcium volume) was tallied in $\mathrm{mm}^{3}$ by CTA analysis, and the patients were stratified into two groups by their calcium volume percentage [(calcium volume/vessel volume)*100]: the non-calcified plaque (NCP) group (calcium volume $\%=0$ ) and the calcified plaque $(\mathrm{CP})$ group (calcium volume $\%>0$ ). Subject characteristics of the two groups are summarized in Table 1. Patients in the CP group, compared with those in the NCP group, were prevalently male $(p=0.006)$, significantly older $(p=0.0002)$ and had a higher Framingham Risk Score $(p<0.0001)$. Moreover they had a higher prevalence of hypertension $(p<0.0001)$, previous myocardial infarction $(p=0.02)$, stenosis $\geq 50 \%$ in at least one coronary artery segment $(p<0.0001)$, hyperlipidemia $(p=0.001)$ and a lower prevalence of smoking $(p=$ $0.03)$.

As shown in Table 2, measurements of vessel (wall plus lumen) and lumen volume did not differ between NCP and CP groups; however, in the CP group, patients with at least one-vessel stenosis $\geq 50 \%$ had a total lumen volume \% significantly lower than in patients without stenosis $(56.6 \pm 11$ vs $61.2 \pm 12$, $p=$ $0.049)$, while a significantly higher total plaque volume $\%(43.12 \pm 11.1$ vs $38.3 \pm 12, p=0.048)$ (Table 2). As expected, all patients with stenosis $\geq 50 \%(n=46)$ had a total plaque burden $\%$ more elevated than 
Table 1. Demographic and clinical characteristics of the study population

\begin{tabular}{|c|c|c|c|}
\hline & NCP group $(n=65)$ & CP group $(n=62)$ & $p$ value \\
\hline Age (yrs) & $58.9 \pm 11.9$ & $66.6 \pm 10.7$ & 0.0002 \\
\hline Men, n (\%) & $34(52)$ & $47(76)$ & 0.006 \\
\hline BMI, $\mathrm{kg} / \mathrm{m}^{2}$ & $26.9 \pm 4$ & $26.6 \pm 2.9$ & 0.66 \\
\hline Hypertension, n (\%) & $11(17)$ & $39(63)$ & $<0.0001$ \\
\hline Diabetes mellitus, n (\%) & $6(9)$ & $10(16)$ & 0.24 \\
\hline Hyperlipidemia, n (\%) & $16(25)$ & $33(53)$ & 0.001 \\
\hline Smoking, n (\%) & $16(25)$ & $6(10)$ & 0.03 \\
\hline Family history of CAD, n (\%) & $41(63)$ & $38(61)$ & 0.83 \\
\hline Previous myocardial infarction, $\mathrm{n}(\%)$ & $2(3)$ & $9(14)$ & 0.02 \\
\hline Stenosis $\geq 50 \%, \mathrm{n}(\%)$ & $8(12)$ & $38(61)$ & $<0.0001$ \\
\hline Framingham risk score & $9.65(5.2-17.34)$ & $16.15(12.1-24.18)$ & $<0.0001$ \\
\hline \multicolumn{4}{|l|}{ Laboratory profile } \\
\hline Glucose (mg/dL) & $106.8 \pm 20.7$ & $100.8 \pm 24.9$ & 0.37 \\
\hline Cholesterol (mg/dL) & $197.4 \pm 46.5$ & $187 \pm 42$ & 0.22 \\
\hline LDL Cholesterol (mg/dL) & $126 \pm 39.7$ & $119.8 \pm 43.8$ & 0.41 \\
\hline HDL Cholesterol (mg/dL) & $46.3 \pm 14.2$ & $46.4 \pm 14$ & 0.97 \\
\hline Triglycerides (mg/dL) & $124(90-150)$ & $106(83.5-140)$ & 0.75 \\
\hline Creatinine (mg/dL) & $0.9(0.8-1.09)$ & $0.99(0.8-1.15)$ & 0.48 \\
\hline sRAGE (pg/mL) & $1115(786-1422)$ & $1038(860-1217)$ & 0.95 \\
\hline
\end{tabular}

Data are given as mean \pm SD, number (\%) or median (interquartile range). NCP group: non-calcified plaque group; CP group: calcified plaque group; $\mathrm{BMI}=$ body mass index; $\mathrm{CAD}=$ coronary artery disease; $\mathrm{LDL}=$ low density lipoprotein; $\mathrm{HDL}=$ high density lipoprotein; SRAGE $=$ soluble receptor for advanced glycation end-products.

Table 2. Vessel characteristics

\begin{tabular}{|c|c|c|c|c|c|c|}
\hline & \multicolumn{3}{|c|}{ NCP group } & \multicolumn{3}{|c|}{$\mathrm{CP}$ group } \\
\hline $\begin{array}{l}\text { Vessel volume } \\
\left(\mathrm{mm}^{3}\right)\end{array}$ & $\begin{array}{c}5235 \\
(4041-6393)\end{array}$ & $\begin{array}{c}4513 \\
(3205-5168)\end{array}$ & $\begin{array}{c}5257 \\
(4139-6706)\end{array}$ & $\begin{array}{c}5172 \\
(3930-6211)\end{array}$ & $\begin{array}{c}5180 \\
(3694-6526)\end{array}$ & $\begin{array}{c}5129 \\
(4270-6057)\end{array}$ \\
\hline $\begin{array}{l}\text { Total lumen volume } \\
\left(\mathrm{mm}^{3}\right)\end{array}$ & $\begin{array}{c}2959 \\
(2516-3978)\end{array}$ & $\begin{array}{c}2676 \\
(1748-2963)\end{array}$ & $\begin{array}{c}3094 \\
(2552-4146)\end{array}$ & $\begin{array}{c}2691 \\
(2388-3772)\end{array}$ & $\begin{array}{c}2672 \\
(2250-3741)\end{array}$ & $\begin{array}{c}3025 \\
(2470-3786)\end{array}$ \\
\hline $\begin{array}{l}\text { Total plaque volume } \\
\left(\mathrm{mm}^{3}\right)\end{array}$ & $\begin{array}{c}2000 \\
(1485-2490)\end{array}$ & $\begin{array}{c}1979 \\
(1093-2205)\end{array}$ & $\begin{array}{c}2059 \\
(1505-2602)\end{array}$ & $\begin{array}{c}2166 \\
(1475-2920)\end{array}$ & $\begin{array}{c}2381 \\
(1513-3006)\end{array}$ & $\begin{array}{c}2054 \\
(1356-2433)\end{array}$ \\
\hline $\begin{array}{l}\text { Total lumen volume } \\
(\%)\end{array}$ & $59.9 \pm 9.6$ & $56.6 \pm 8.99$ & $60.4 \pm 9.6$ & $58.3 \pm 11.6$ & $56.6 \pm 11$ & $61.2 \pm 12^{*}$ \\
\hline $\begin{array}{l}\text { Total plaque volume } \\
(\%)\end{array}$ & $39.6 \pm 9.5$ & $42.8 \pm 9.1$ & $39.1 \pm 9.5$ & $41.3 \pm 11.6$ & $43.12 \pm 11.1$ & $38.3 \pm 12^{* *}$ \\
\hline
\end{tabular}

NCP group: non-calcified plaque group; CP group: calcified plaque group. Data are given as mean \pm SD or median (interquartile range). ${ }^{*} p=0.049,{ }^{* *} p=0.048$ between patients with stenosis $\geq 50 \%$ and patients without stenosis $\geq 50 \%$.

patients without stenosis $(43.0 \pm 10.7$ vs $38.9 \pm 10.3$, $p=0.032$ ) while they had a total lumen volume $\%$ lower than patients without stenosis $(56.5 \pm 10.7$ vs $60.6 \pm 10.3, p=0.036$ ).

Using univariate linear regression analysis, in the whole study population, the plaque burden was inversely associated with plasma levels of sRAGE ( $\beta=$ $-0.201, p=0.02)$ and HDL cholesterol $(\beta=-0.287$, $p=0.014$ ), while it was positively associated with male gender $(\beta=0.264, p=0.0027)$, hyperlipidemia $(\beta=$ $0.271, p=0.0025)$ and creatinine (Table 3). Regression analysis, performed in two separate groups, 
Table 3. Simple regression analysis with percentage of plaque burden as dependent variable

\begin{tabular}{|c|c|c|c|c|c|c|}
\hline & \multicolumn{2}{|c|}{ All patients $(n=127)$} & \multicolumn{2}{|c|}{ NCP group $(n=65)$} & \multicolumn{2}{|c|}{$\mathrm{CP}$ group $(n=62)$} \\
\hline & $\beta$ & $p$ value & $\beta$ & $p$ value & $\beta$ & $p$ value \\
\hline Age & -0.053 & 0.55 & -0.122 & 0.33 & -0.049 & 0.702 \\
\hline Male & 0.264 & 0.0026 & 0.317 & 0.01 & 0.198 & 0.124 \\
\hline BMI & 0.134 & 0.14 & 0.026 & 0.84 & 0.28 & 0.029 \\
\hline Hypertension & 0.076 & 0.403 & -0.046 & 0.72 & 0.105 & 0.42 \\
\hline Diabetes mellitus & 0.095 & 0.286 & 0.196 & 0.118 & 0.015 & 0.909 \\
\hline Hyperlipidemia & 0.271 & 0.0025 & 0.237 & 0.064 & 0.292 & 0.022 \\
\hline Smoking & -0.043 & 0.63 & 0.013 & 0.915 & -0.082 & 0.529 \\
\hline Family history of CAD & -0.032 & 0.72 & -0.12 & 0.340 & 0.045 & 0.730 \\
\hline Anti-thrombotic therapy & -0.004 & 0.96 & -0.127 & 0.998 & 0.088 & 0.49 \\
\hline Creatinine & 0.200 & 0.027 & 0.26 & 0.041 & 0.150 & 0.26 \\
\hline HDL Cholesterol & -0.287 & 0.0013 & -0.368 & 0.003 & -0.223 & 0.087 \\
\hline sRAGE & -0.201 & 0.02 & -0.378 & 0.0019 & -0.034 & 0.794 \\
\hline
\end{tabular}

NCP group: non-calcified plaque group; CP group: calcified plaque group.

Table 4. Multiple regression analysis with percentage of plaque burden as dependent variable

\begin{tabular}{|c|c|c|c|c|c|c|}
\hline & \multicolumn{2}{|c|}{ All patients $(n=127)$} & \multicolumn{2}{|c|}{ NCP group $(n=65)$} & \multicolumn{2}{|c|}{$\mathrm{CP}$ group $(n=62)$} \\
\hline & $\beta$ & $p$ value & $\beta$ & $p$ value & $\beta$ & $p$ value \\
\hline Age & 0.064 & 0.582 & 0.061 & 0.717 & -0.047 & 0.78 \\
\hline Male & 0.179 & 0.100 & 0.216 & 0.200 & 0.101 & 0.55 \\
\hline BMI & 0.028 & 0.762 & -0.141 & 0.293 & 0.279 & 0.068 \\
\hline Hypertension & -0.054 & 0.603 & -0.111 & 0.439 & 0.105 & 0.51 \\
\hline Diabetes mellitus & 0.053 & 0.568 & 0.051 & 0.709 & -0.21 & 0.88 \\
\hline Hyperlipidemia & 0.223 & 0.032 & 0.207 & 0.165 & 0.234 & 0.13 \\
\hline Smoking & -0.112 & 0.25 & -0.141 & 0.293 & -0.33 & 0.83 \\
\hline Family history of CAD & 0.006 & 0.95 & 0.003 & 0.979 & -0.012 & 0.94 \\
\hline Anti-thrombotic therapy & -0.103 & 0.32 & -0.242 & 0.111 & 0.0002 & 0.998 \\
\hline Creatinine & 0.145 & 0.118 & 0.087 & 0.508 & 0.077 & 0.604 \\
\hline HDL Cholesterol & -0.123 & 0.274 & -0.136 & 0.457 & -0.169 & 0.324 \\
\hline sRAGE & -0.149 & 0.106 & -0.358 & 0.0115 & 0.013 & 0.930 \\
\hline
\end{tabular}

NCP group: non-calcified plaque group; CP group: calcified plaque group.

revealed an inverse association between total plaque burden and both sRAGE levels $(\beta=-0.378$, $p=0.0019)$ and HDL cholesterol $(\beta=-0.368$, $p=0.003$ ) only in the NCP group (Table 3). In addition, in the NCP group, the percentage of total plaque burden was directly associated with creatinine $(\beta=$ $0.258, p=0.041)$ and with male gender $(\beta=0.317$, $p=0.01$ ) (Table 3). In the CP group, the percentage of total plaque burden was significantly associated with BMI $(\beta=0.284, p=0.03)$ and hyperlipidemia $(\beta=0.292, p=0.02)$. Multivariate linear regression analysis showed that in the NCP group, the total plaque burden remained only significantly associated with sRAGE after adjusting for confounding factors and pre-defined variables (Table 4).

Regarding the association between sRAGE and the variables indicated in Table $\mathbf{1}$, we found a positive association only with HDL cholesterol $(\beta=0.336$, $p=0.0073$ ) in the NCP group (Fig. 1), while no association between sRAGE and diabetes presence was found either in the entire population of the study or in the two separate groups (data not shown). The lack of association between sRAGE levels and diabetes may be due to the low prevalence of diabetics in the enrolled population $(12.5 \%)$ and to the anti-diabetic treatments. 


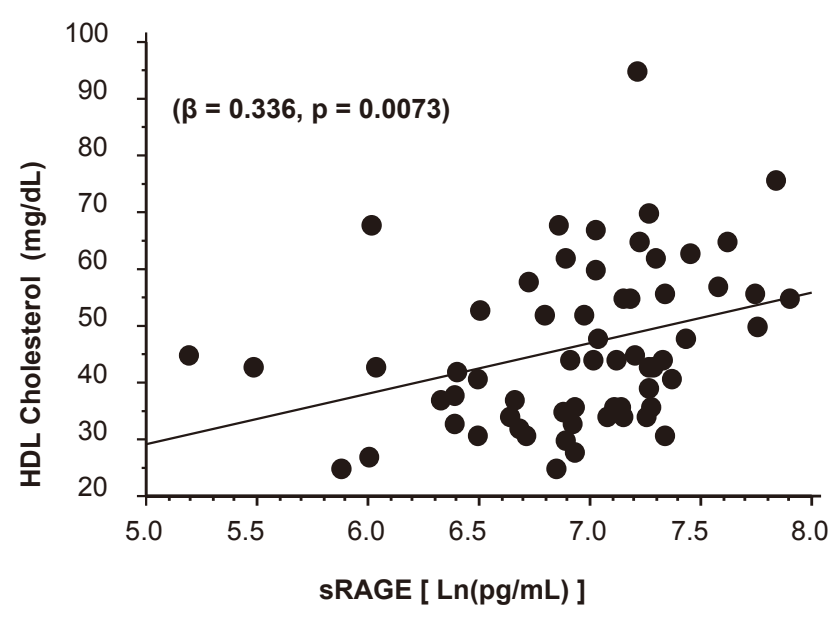

Fig. 1. Correlation between sRAGE and HDL cholesterol levels in NCP group.

\section{Discussion}

Our study was designed to examine the relationship of sRAGE with plaque composition and plaque burden through non-invasive CTA assessment. We found that sRAGE plasma levels were inversely associated with total plaque burden in all patients and remained strongly associated in the sub-group of patients without coronary calcification after adjusting for confounding variables. Although previous studies have shown an independent association between low levels of sRAGE and the presence of $\mathrm{CAD}^{6,7)}$, this is the first study that shows an inverse association between sRAGE and both coronary atherosclerotic plaque burden and composition detected by CTA. This association is relevant because it is mainly significant in patients with NCP, suggesting a role of sRAGE in the early pathogenesis of atherosclerosis and possibly in the progression and vulnerability of coronary atherosclerotic plaque ${ }^{17,18)}$. Although the exact mechanism of the inverse association between sRAGE and plaque burden remains to be determined, we believe that this biomarker may provide useful information on plaque activity or, better still, of ligand-RAGE hyperactivity associated with the vascular disease state. In other words, the balance among the levels of RAGE ligands, cell-surface RAGE and sRAGE may represent a complex dynamic system and the down-regulation of this latter and the up-regulation of both tissue RAGE and RAGE ligands could have important consequences for the function of endothelial cells, monocytes, macrophages, smooth muscle cells and platelets, thus modulating the initiation and progression of atherosclerosis.
Vascular calcification is age-dependent and is a regulated/active process which could depend also on the ligand-RAGE axis ${ }^{19)}$. The Dallas Heart Study (2571 subjects, mean age $44 \pm 10$ years), reported an inverse graded association between sRAGE quartiles and coronary artery calcium (by electron-beam $\mathrm{CT}{ }^{20)}$. Instead, in our study, we found no association between sRAGE and calcium volume (data not shown). This discrepancy could be due to the fact that our patients were older $(66.6 \pm 10.7$ versus $44 \pm 10$ years) and had a higher degree of CAD severity and calcification than the Dallas Heart Study population. Therefore the association between sRAGE and plaque burden and/or calcification would seem to be lacking in elderly patients with advanced atherosclerotic plaques, characterized by extensive calcification. At this stage of atherosclerosis the inflammatory processes involving the RAGE-ligand axis and sRAGE should be shut down. Hence, we can assume that sRAGE is promising as a potential therapeutic target for the treatment and prevention of atherosclerosis.

We found also a positive correlation between sRAGE and HDL cholesterol in the NCP group. We are unable to explain the significance of this relationship but it could suggest a potential link between sRAGE and HDL metabolism.

Our study has some limitations. This is a crosssectional study, which limits causal inferences. Also, single measurements of sRAGE and CTA might not be representative of changes in levels over time. In addition, the ELISA kit used detects the total soluble pool of sRAGE and does not distinguish between different sRAGE isoforms. Another limitation is that we measured coronary calcium content from CTA. The presence of contrast medium in CTA images forces reduction of the window of $\mathrm{HU}$ for calcium recognition, causing an underestimation of calcium volume. However, data from our laboratory showed a strong correlation between coronary calcium content measured with the standard calcium scoring method without contrast medium and CTA images $(\mathrm{Y}=1.47+$ $\left.0.128 * \mathrm{X} ; R^{2}=0.874, \mathrm{~F}=660.6, p<0.00001\right)$. Avoiding dedicated CT acquisition for calcium scoring before the angiographic studies allowed about $10 \%$ sparing of the radiation dose.

\section{Conclusion}

In conclusion, this survey of $\mathrm{CAD}$ patients shows that circulating sRAGE levels are associated in an inverse manner with coronary plaque burden in patients without calcifications. These findings are compatible with the hypothesis that high sRAGE may 
act early as a vasculo-protective factor in this population. Longitudinal observations and intervention studies are warranted to establish whether this link is causal in nature.

\section{Acknowledgements}

Funding sources: The study was supported in part by grants from FP7-ICT-2007 (grant agreement 224297, ARTreat). The authors thank the radiology technicians, nurses of the invasive cardiovascular unit and the staff of the clinical laboratory of the Monasterio Foundation for their active collaboration. In addition, we thank Antonella Cecchi for her secretarial assistance and Alison Frank for her assistance in editing the English language.

\section{Conflict of Interest}

No.

\section{References}

1) Bopp C, Bierhaus A, Hofer S, Bouchon A, Nawroth PP, Martin E, Weigand MA: Bench-to-bedside review: The inflammation-perpetuating pattern-recognition receptor RAGE as a therapeutic target in sepsis. Crit Care, 2008; 12: 201

2) Basta G: Receptor for advanced glycation endproducts and atherosclerosis: From basic mechanisms to clinical implications. Atherosclerosis, 2008; 196: 9-21

3) Yan SF, D’Agati V, Schmidt AM, Ramasamy R: Receptor for Advanced Glycation Endproducts (RAGE): a formidable force in the pathogenesis of the cardiovascular complications of diabetes \& aging. Curr Mol Med, 2007; 7: 699-710

4) Santilli F, Vazzana N, Bucciarelli LG, Davi G: Soluble forms of RAGE in human diseases: clinical and therapeutical implications. Curr Med Chem, 2009; 16: 940-952

5) Ramasamy R, Yan SF, Schmidt AM: RAGE: therapeutic target and biomarker of the inflammatory response--the evidence mounts. J Leukoc Biol, 2009; 86: 505-512

6) Falcone C, Emanuele E, D’Angelo A, Buzzi MP, Belvito C, Cuccia M, Geroldi D: Plasma levels of soluble receptor for advanced glycation end products and coronary artery disease in nondiabetic men. Arterioscler Thromb Vasc Biol, 2005; 25: 1032-1037

7) Chiang KH, Huang PH, Huang SS, Wu TC, Chen JW, Lin SJ: Plasma levels of soluble receptor for advanced glycation end products are associated with endothelial function and predict cardiovascular events in nondiabetic patients. Coron Artery Dis, 2009; 20: 267-273

8) Koyama H, Shoji T, Yokoyama H, Motoyama K, Mori K, Fukumoto S, Emoto M, Shoji T, Tamei H, Matsuki H, Sakurai S, Yamamoto Y, Yonekura H, Watanabe T, Yamamoto H, Nishizawa Y: Plasma level of endogenous secretory RAGE is associated with components of the meta- bolic syndrome and atherosclerosis. Arterioscler Thromb Vasc Biol, 2005; 25: 2587-2593

9) Basta G, Leonardis D, Mallamaci F, Cutrupi S, Pizzini P, Gaetano L, Tripepi R, Tripepi G, De Caterina R, Zoccali C: Circulating soluble receptor of advanced glycation end product inversely correlates with atherosclerosis in patients with chronic kidney disease. Kidney Int, 2010; 77: 225-231

10) Hausleiter J, Meyer T, Hadamitzky M, Kastrati A, Martinoff S, Schomig A: Prevalence of noncalcified coronary plaques by 64-slice computed tomography in patients with an intermediate risk for significant coronary artery disease. J Am Coll Cardiol, 2006; 48: 312-318

11) Jinzaki M, Yamada M, Sato K, Tanami Y, Anzai T, Sasaki K, Kuribayashi S: Overview image of the lumen and vessel wall in coronary CT angiography. Circ J, 2008; 72: 671-673

12) Matsunaga E, Takaya N, Yokoyama T, Akimoto Y, Miyauchi K, Daida H: Relationship between coronary artery wall thickness measured by 64-slice multidetector computed tomography and cardiovascular risk factors. Circ J, 2009; 73: 681-685

13) Achenbach S, Moselewski F, Ropers D, Ferencik M, Hoffmann U, MacNeill B, Pohle K, Baum U, Anders K, Jang IK, Daniel WG, Brady TJ: Detection of calcified and noncalcified coronary atherosclerotic plaque by contrastenhanced, submillimeter multidetector spiral computed tomography: a segment-based comparison with intravascular ultrasound. Circulation, 2004; 109: 14-17

14) Hendel RC, Patel MR, Kramer CM, Poon M, Hendel RC, Carr JC, Gerstad NA, Gillam LD, Hodgson JM, Kim RJ, Kramer CM, Lesser JR, Martin ET, Messer JV, Redberg RF, Rubin GD, Rumsfeld JS, Taylor AJ, Weigold WG, Woodard PK, Brindis RG, Hendel RC, Douglas PS, Peterson ED, Wolk MJ, Allen JM, Patel MR: ACCF/ ACR/SCCT/SCMR/ASNC/NASCI/SCAI/SIR 2006 appropriateness criteria for cardiac computed tomography and cardiac magnetic resonance imaging: a report of the American College of Cardiology Foundation Quality Strategic Directions Committee Appropriateness Criteria Working Group, American College of Radiology, Society of Cardiovascular Computed Tomography, Society for Cardiovascular Magnetic Resonance, American Society of Nuclear Cardiology, North American Society for Cardiac Imaging, Society for Cardiovascular Angiography and Interventions, and Society of Interventional Radiology. J Am Coll Cardiol, 2006; 48: 1475-1497

15) Wilson PW, D’Agostino RB, Levy D, Belanger AM, Silbershatz H,Kannel WB: Prediction of coronary heart disease using risk factor categories. Circulation, 1998; 97: 1837-1847

16) Arai $K$, Ishii $H$, Amano T, Uetani T, Nanki M, Marui N, Kato M, Yokoi K, Ando H, Kumagai S, Harada K, Yoshikawa D, Ohshima S, Matsubara T, Murohara T: Volumetric analysis of coronary plaque characterization in patients with metabolic syndrome using 64-slice multi-detector computed tomography. Circ J, 2010; 74: 2146-2151

17) Stary HC, Chandler AB, Dinsmore RE, Fuster V, Glagov S, Insull W Jr, Rosenfeld ME, Schwartz CJ, Wagner WD, Wissler RW: A definition of advanced types of atheroscle- 
rotic lesions and a histological classification of atherosclerosis. A report from the Committee on Vascular Lesions of the Council on Arteriosclerosis, American Heart Association. Circulation, 1995; 92: 1355-1374

18) Hoffmann U, Moselewski F, Nieman K, Jang IK, Ferencik M, Rahman AM, Cury RC, Abbara S, Joneidi-Jafari H, Achenbach S, Brady TJ: Noninvasive assessment of plaque morphology and composition in culprit and stable lesions in acute coronary syndrome and stable lesions in stable angina by multidetector computed tomography. J Am
Coll Cardiol, 2006; 47: 1655-1662

19) Towler DA: Vascular calcification: it's all the RAGE! Arterioscler Thromb Vasc Biol, 2011; 31: 237-239

20) Lindsey JB, de Lemos JA, Cipollone F, Ayers CR, Rohatgi A, Morrow DA, Khera A, McGuire DK: Association between circulating soluble receptor for advanced glycation end products and atherosclerosis: observations from the Dallas Heart Study. Diabetes Care, 2009; 32: 1218 1220 